\title{
VGOS Intensives Ishioka-Onsala
}

\section{Other Conference Item}

\section{Author(s):}

Haas, Rüdiger; Varenius, Eskil; Diamantidis, Periklis-Konstantinos; Matsumotu, Saho; Schartner, Matthias (D); Nilsson, Tobias

Publication date:

2021

Permanent link:

https://doi.org/10.3929/ethz-b-000483770

Rights / license:

Creative Commons Attribution 4.0 International

Originally published in:

EGUsphere, https://doi.org/10.5194/egusphere-egu21-12916 
EGU21-12916

https://doi.org/10.5194/egusphere-egu21-12916

EGU General Assembly 2021

(c) Author(s) 2021. This work is distributed under

the Creative Commons Attribution 4.0 License.

\section{VGOS Intensives Ishioka-Onsala}

Rüdiger Haas ${ }^{1}$, Eskil Varenius ${ }^{1}$, Periklis-Konstantinos Diamantidis ${ }^{1}$, Saho Matsumotu ${ }^{2}$, Matthias Schartner ${ }^{3}$, and Tobias Nilsson ${ }^{4}$

${ }^{1}$ Chalmers University of Technology, Space, Earth and Environment, Space Geodesy and Geodynamics, Onsala, Sweden (rudiger.haas@chalmers.se)

${ }^{2}$ Geospatial Information Authority of Japan

${ }^{3}$ ETH Zロürich, Institute of Geodesy and Photogrammetry, Zürich, Switzerland

${ }^{4}$ Lantmäteriet - The Swedish mapping, cadastral and land registration authority, Geodetic Infrastructure, Gävle, Sweden

The VLBI Global Observing System (VGOS) is the VLBI contribution to GGOS. During the last years, several VGOS stations have been established, the VGOS observation program has started, and by 2021 VGOS has achieved an operational state involving nine international VGOS stations. Further VGOS stations are currently being installed, so that the number of active VGOS stations will increase drastically in the near future. In the end of 2019 the International VLBI Service for Geodesy and Astrometry (IVS) decided to start a new and so-far experimental VGOS-Intensive series, called VGOS-B, involving Ishioka (Japan) and Onsala (Sweden). Both sites operate modern VGOS stations with 13.2 m diameter radio telescopes, i.e. ISHIOKA (IS) in Japan, and ONSA13NE (OE) and ONSA13SW (OW) in Sweden. In total 12 VGOS-B sessions were observed between December 2019 and February 2020, one every week, in parallel and simultaneously to legacy S/X INT1 Intensive sessions that involve the stations KOKEE (KK) on Hawaii and WETTZELL (WZ) in Germany. These 1-hour long VGOS-B sessions consist of more than fifty radio source observations, resulting in about 1.6 TB of raw data that are collected at each station. The scheduling of the VGOSB sessions was done using VieSched++ and the subsequent steps (correlation, fringe-fitting, database creation) were carried out at the Onsala Space Observatory using DIFX and HOPS. The resulting VGOS databases were analysed with several VLBI analysis software packages, involving nuSolve, $\mathrm{C5++}$ and ASCOT. In this presentation, we give an overview on the VGOS-B series, present our experiences, and discuss the obtained results. The derived UT1-UTC results were compared to corresponding results from standard legacy S/X Intensive sessions (INT1/INT2), as well to the final values of the International Earth Rotation and Reference Frame Service (IERS), provided in IERS Bulletin B.

The VGOS-B series achieve 3-4 times lower formal uncertainties for the UT1-UTC results than standard legacy S/X INT series. Furthermore, the root mean square (RMS) agreement with respect to the IERS Bulletin B is 30-40 \% better for the VGOS-B results than for the INT1/INT2 results. 